\title{
Evaluación de los conocimientos prácticos de los pacientes prevalentes en diálisis peritoneal
}

\author{
Beatriz Peláez Requejo*, Mónica Fernández Pérez*, Miguel Núñez Moral*, Isabel González Díaz*, Alejan- \\ dra Méndez González*, Aurora Quintana Fernández**
}

*Enfermeros/as, **Auxiliar de Enfermería, Unidad de Diálisis Peritoneal del Hospital Universitario Central de Asturias. Oviedo

\section{Resumen}

La fase de entrenamiento, en la que se dota a pacientes y cuidadores de los conocimientos y habilidades necesarios para realizar su autotratamiento, es vital.

El objetivo del estudio fue evaluar los conocimientos prácticos de los pacientes de nuestra Unidad mediante la realización de un intercambio manual y como consecuencia de éste, valorar la necesidad de establecer un programa de reentrenamiento reglado.

Se realizó un estudio observacional, transversal entre Febrero y Abril de 2013, con pacientes prevalentes, mayores de edad, con más de un mes en técnica domiciliaria. Se diseñó un test de evaluación de 23 ítems que recogía los pasos de un intercambio manual y la administración de medicación intraperitoneal; la valoración la realizó un observador imparcial durante una revisión rutinaria del paciente.

Se recogieron variables epidemiológicas, clínicas y nivel de estudios. El análisis estadístico se realizó con el paquete informático SPSS 20.0.

Se evaluaron 62 pacientes, 59 con Enfermedad Renal Crónica (24 en Diálisis Peritoneal Continua Ambulatoria y 35 en Diálisis Peritoneal Automatizada) y 3 con Insuficiencia Cardiaca Congestiva. EI tiempo medio en técnica fue $19 \pm 20$ meses en ma-

\section{Correspondencia: \\ Beatriz Peláez Requejo \\ Camino de la Iglesia, $20,2^{\circ} \mathrm{G}$ \\ 33690 Lugo de Llanera. Asturias \\ E-mail: beatrizpelaezr@yahoo.es}

nual, $24 \pm 27$ en automática y $8 \pm 4$ en ultrafiltración peritoneal.

La media de aciertos fue de $18 \pm 3$, sin diferencias en cuanto a las variables analizadas.

Nuestros pacientes están, en general, bien formados, con variaciones individuales no dependientes de edad, sexo, educación o tiempo en la técnica.

Consideramos que un buen entrenamiento no es suficiente, siendo pertinente establecer un programa de reentrenamientos periódicos y personalizados.

PALABRAS CLAVE:

- DIÁLISIS PERITONEAL

- AUTOCUIDADO

- ENTRENAMIENTO

- REENTRENAMIENTO

Assessment of practical knowledge of prevalent peritoneal dialysis patients

\section{Abstract}

The training phase, when patients and carers are equipped with the knowledge and skills necessary to carry out self-treatment, is vital.

The aim of the study was to assess the practical knowledge of patients in our Unit by carrying out a manual exchange and, as a result, assessing the need to establish a regulated retraining programme.

An observational, transversal study was carried out between February and April 2013, with prevalent 
patients, aged over 18, who had spent more than one month on home technique. An assessment test with 23 items was designed, which reflected the steps of a manual exchange and intraperitoneal medication administration; the assessment was performed by an impartial observer during a routine patient check-up.

Epidemiological, clinical and level of education variables were collected. The statistical analysis was performed using the computer package SPSS 20.0.

A total of 62 patients were assessed, 59 with Chronic Kidney Disease (24 on Continuous Ambulatory Peritoneal Dialysis and 35 on Automated Peritoneal Dialysis) and 3 with Congestive Heart Failure. The average time using the technique was $19 \pm 20$ months in manual, $24 \pm 27$ in automatic and $8 \pm 4$ in peritoneal ultrafiltration.

The average number of correct answers was $18 \pm 3$, with no difference in relation to the variables analysed.

In general, our patients are well-trained, with individual variations that do not depend on age, sex, education or time using the technique.

We consider that good training is not sufficient, and that regular, personalized retraining programmes should be established.

\section{KEY WORDS}

- PERITONEAL DIALYSIS

- SELF-CARE

- TRAINING

- RETRAINING

\section{Introducción}

La autonomía es uno de los principales beneficios de la técnica de Diálisis Peritoneal (DP) y lo que hace que muchos de los pacientes que deben iniciar tratamiento renal sustitutivo la elijan como modalidad dialítica. Este concepto va íntimamente ligado al de autotratamiento, así que, la fase de entrenamiento, en la que se dota a pacientes y cuidadores de los conocimientos y habilidades necesarios para realizarlo correctamente en su domicilio, es vital y está suficientemente acreditado que tiene enorme influencia sobre el éxito de la técnica y la prevención de las complicaciones (Evidencia C) ) $^{1,2,3,4}$.

El entrenamiento debe cumplir esa premisa pero debemos tener en cuenta que depende de muchos factores, entre ellos, el nivel de conocimientos previos, el nivel de ansiedad durante el mismo y el estado urémico de los pacientes. Todos condicionaran básicamente el número de visitas necesarias para completarlo.

El proceso de entrenamiento en nuestra Unidad se inicia de forma temprana tras la implantación del catéter peritoneal, se realiza en el Hospital y se distribuye en 2 o 3 sesiones semanales de aproximadamente, una hora de duración; el tiempo medio de entrenamiento ronda el mes, aunque a veces es necesario prolongarlo para asegurar la correcta realización del autocuidado y siempre se hace en relación 1:1 (instructor/paciente), lo que permite que sea totalmente individualizado y adaptado a las dificultades de aprendizaje de cada paciente.

Esta formación, que consume gran cantidad de tiempo, al igual que en la mayoría de los centros, corre a cargo del equipo de Enfermería de la Unidad, formado por cuatro enfermeras y una supervisora, con dedicación completa y exclusiva (no rotamos por otras Unidades) a la DP. Estas enfermeras han recibido formación específica en Nefrología y tras su incorporación a la Unidad, acompañan durante un periodo de tiempo, a otra de más antigüedad durante al menos, el entrenamiento íntegro de un paciente. El hecho de que los conocimientos más especíicos sean proporcionados por otra compañera es una práctica habitual en las Unidades de todo el mun$\mathrm{do}^{4,5,6}$.

Pero un buen entrenamiento inicial no es suficiente y la experiencia y los estudios señalan que con el paso del tiempo y en parte debido a la cronicidad del tratamiento, se modifican inconscientemente las pautas de actuación. Varios autores hacen referencia además de a la "curva del olvido" que explica cómo se va perdiendo la información y la importancia del repaso y la repetición, a la creación de una "falsa memoria" por la que el mensaje original se va transformando y nos hace creer que la forma en que hacemos las cosas es la forma en la que se nos enseñóo, $4,5,6,7,8$.

Nuestro objetivo es evaluar la correcta realización de la técnica por parte de nuestros pacientes y en consecuencia, la necesidad de establecer un programa de reentrenamiento reglado. 


\section{Material y métodos}

Se realizó un estudio observacional, transversal entre Febrero y Abril de 2013 en la Unidad de Diálisis Peritoneal del Hospital U. Central de Asturias, donde se evaluó a pacientes prevalentes, mayores de 18 años y con una permanencia superior a un mes realizando la técnica en su domicilio.

Se diseñó un test de evaluación de 23 ítems (tabla 1) que recogía los pasos a seguir durante la realización de un intercambio manual y la administración de medicación intraperitoneal.

\section{IDENTIFICACIÓN:}

\begin{tabular}{|c|c|c|c|}
\hline LISTA DE VERIFICACIÓN & SI & NO & COMENTARIOS \\
\hline 1. Lava las manos & & & \\
\hline 2. Limpia la mesa & & & \\
\hline 3. Prepara los productos necesarios & & & \\
\hline $\begin{array}{l}\text { 4. Retira la sobrebolsa de la bolsa de } \\
\text { líquido caliente }\end{array}$ & & & \\
\hline $\begin{array}{l}\text { 5. Comprueba concentración, fecha } \\
\text { de caducidad, que esté clara y que } \\
\text { no tenga fugas }\end{array}$ & & & \\
\hline 6. Se pone mascarilla & & & \\
\hline $\begin{array}{l}\text { 7. Saca el prolongador cuidadosa- } \\
\text { mente, sin tirones }\end{array}$ & & & \\
\hline 8. Lava las manos 3 minutos & & & \\
\hline $\begin{array}{l}\text { 9. Se frota las manos con desinfec- } \\
\text { tante }\end{array}$ & & & \\
\hline 10. Conexión & & & \\
\hline $\begin{array}{l}\text { 11. Procede al drenado el tiempo ne- } \\
\text { cesario (15-20 } \mathrm{min})\end{array}$ & & & \\
\hline 12. Purgado de líneas & & & \\
\hline 13. Infusión & & & \\
\hline 14. Cierra prolongador y líneas & & & \\
\hline 15. Prepara el tapón nuevo & & & \\
\hline $\begin{array}{l}\text { 16. Se frota las manos con desinfec- } \\
\text { tante }\end{array}$ & & & \\
\hline 17. Desconexión & & & \\
\hline $\begin{array}{l}\text { 18. Comprueba aspecto del líquido } \\
\text { drenado }\end{array}$ & & & \\
\hline 19. Pesa el líquido drenado & & & \\
\hline 20. Apunta en la gráfica & & & \\
\hline $\begin{array}{l}\text { 21. Desecha el equipo y el líquido dre- } \\
\text { nado }\end{array}$ & & & \\
\hline 22. Se lava las manos & & & \\
\hline 23. Administra heparina & & & \\
\hline
\end{tabular}

Tabla 1. Lista de Verificación. REENTRENAMIENTO: EVALUACIÓN INTERCAMBIO MANUAL.

Los pacientes realizaron el intercambio manual real 0 con delantal de simulación, en el hospital y en el contexto de una revisión rutinaria; fueron supervisados y corregidos inmediatamente si se producía algún error. La evaluación la realizó un mismo observador imparcial, una enfermera de la Unidad que no había participado en el proceso de entrenamiento inicial de ninguno de ellos.

Previamente se obtuvieron los consentimientos informados de cada uno de los pacientes y la aprobación del estudio por parte del Comité de Ética del hospital.

Se creó una base de datos electrónica que incluía variables epidemiológicas, clínicas y nivel de estudios. Como variables epidemiológicas incluimos la edad y el sexo, como variables clínicas el tiempo en técnica desde el inicio en domicilio y las diferentes modalidades, Diálisis Peritoneal Continua Ambulatoria (CAPD), Diálisis Peritoneal Automatizada (APD) y Ultrafiltración Peritoneal (UP); además, clasificamos el nivel de estudios en primarios, secundarios y universitarios.

Las variables continuas se expresaron como media y desviación típica si seguían una distribución normal y como mediana y rango intercuartílico cuando no la tenían; previamente se había empleado el test de Kolmogorov Smirnov para ver si seguían una distribución normal. Las variables categóricas se expresaron como porcentaje. Para las correlaciones univariadas, se empleó la $r$ de Pearson. Para conocer los mejores predictores de respuesta al tratamiento, se aplicó un modelo de regresión lineal. Se utilizó el paquete estadístico SPSS ${ }^{\circledR} 20.0$ para Windows (SPSS Inc, Chicago, IL) para el análisis de los resultados.

\section{Resultados}

Se evaluaron 62 pacientes, 59 con Enfermedad Renal Crónica (ERC) de los cuales 24 estaban en programa de DPCA y 35 en DPA y solo 3 pacientes con Insuficiencia Cardiaca Congestiva (ICC). En el gráfico 1 se describe

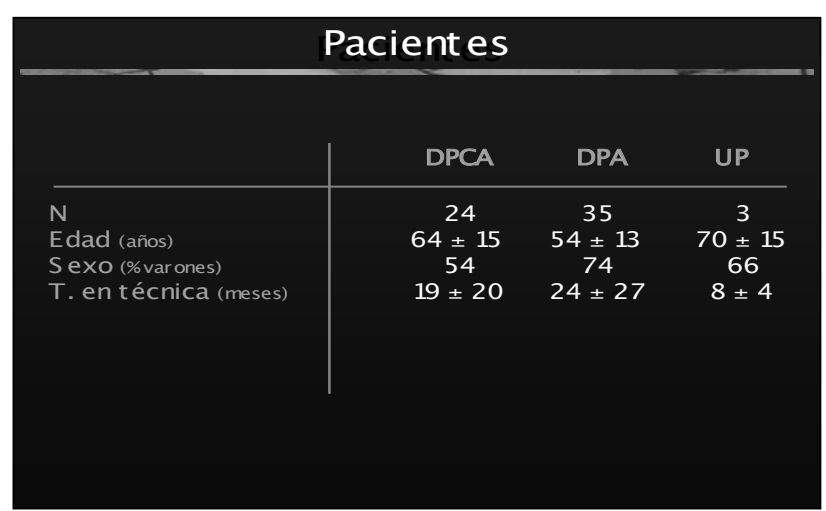

Gráfico 1. Distribución de pacientes. 
su distribución por edad, sexo, modalidad y tiempo en técnica.

La media de aciertos de los ítems analizados fue de 18 \pm 3 , lo que supuso el $75.8 \%$ con un rango entre 43-96 $\%$. Analizando la correcta ejecución del procedimiento, señalamos el porcentaje de buena práctica de algunos puntos clave: colocación de mascarilla $79 \%$, lavado quirúrgico de manos correcto y en el momento adecuado $65 \%$, desinfección pre-conexión $85 \%$, conexión $85 \%$, desinfección pre-desconexión $61 \%$, desconexión $98 \%$, comprobación del aspecto del efluente $100 \%$ y administración de medicación intraperitoneal (1cc de heparina sódica) $69 \%$.

No hubo diferencias en las respuestas acertadas en cuanto a sexo, edad, modalidad, tiempo en técnica domiciliaria o nivel de estudios. Ninguna de las variables analizadas fue predictor independiente del número de aciertos.

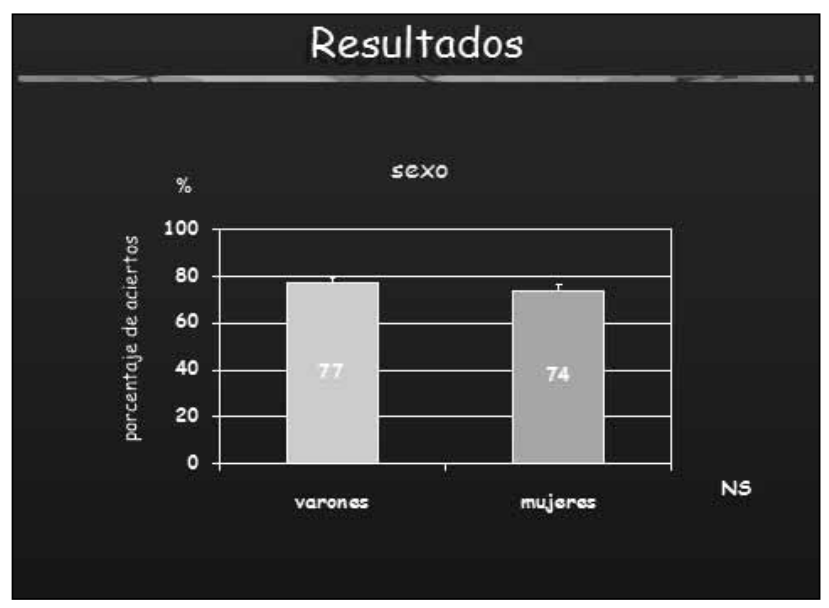

Gráfico 2. Aciertos según sexo.

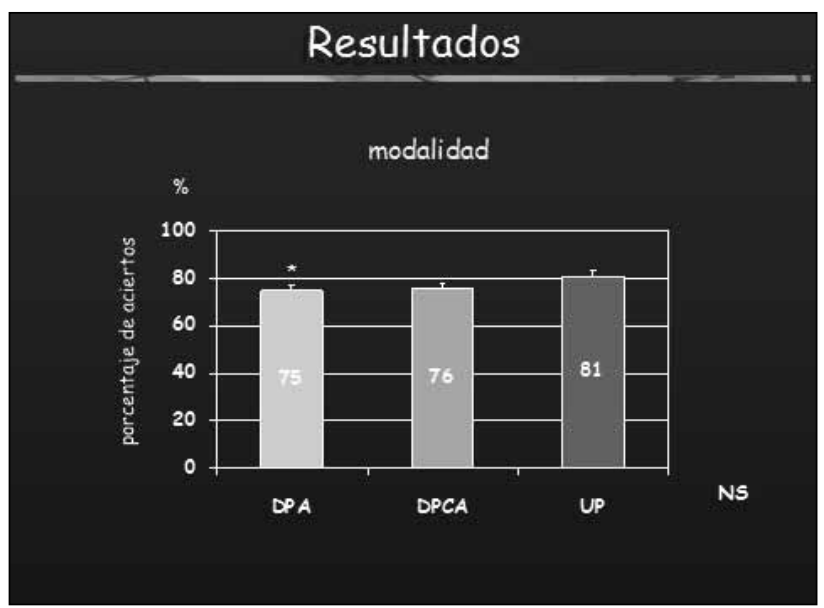

Gráfico 3. Aciertos según modalidad.

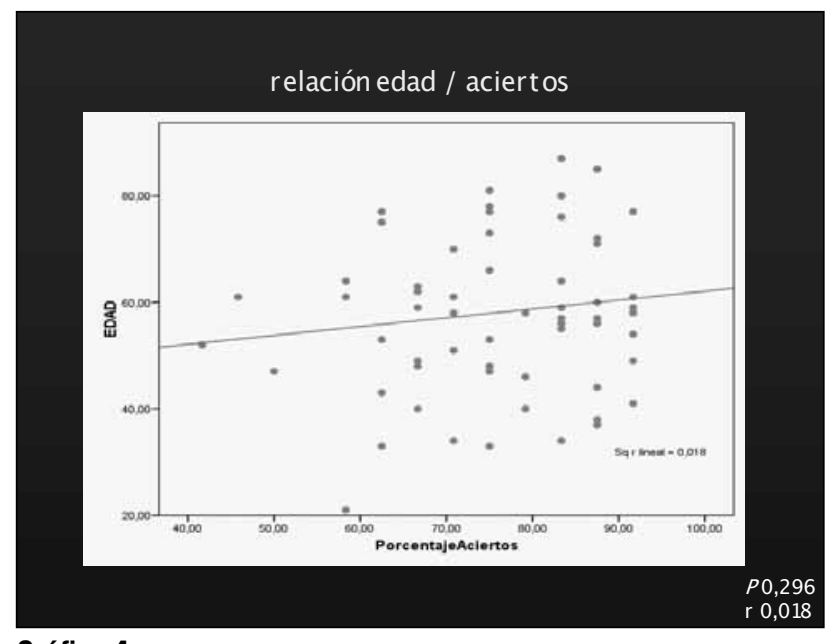

Gráfico 4.

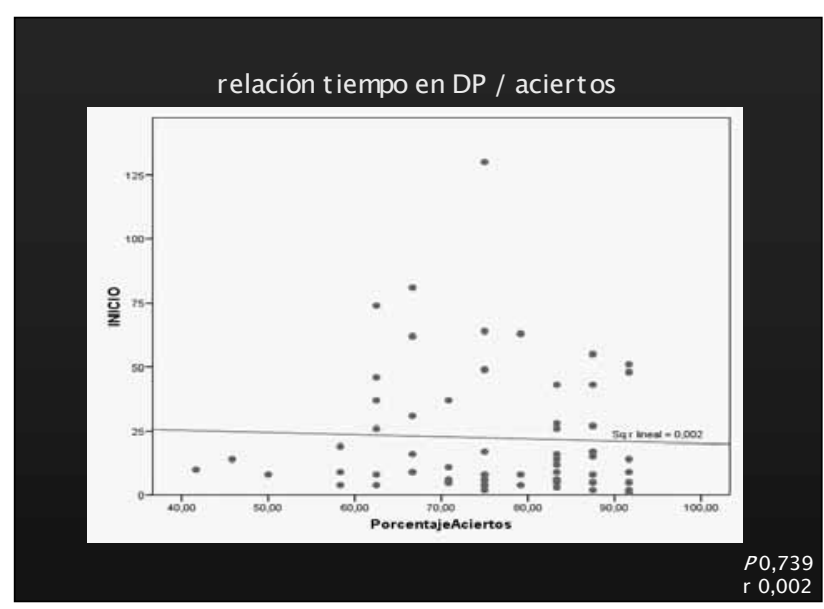

Gráfico 5.

\section{Discusión}

La prevención de las potenciales complicaciones infecciosas inherentes a la técnica de la DP es una preocupación desde sus inicios. Al igual que la profilaxis antibiótica previa a la implantación del catéter o el control y tratamiento de los portadores nasales, la correcta realización de la técnica es un pilar fundamental ${ }^{7,9}$.

Algunos estudios anteriores analizan esta misma cuestión y plantean la conveniencia de reciclar o reentrenar a los pacientes como mejor alternativa para tratar de asegurarla.

Russo et al, en el artículo publicado en 2006, ya pregunta por qué y cuándo es necesario el reentrenamiento después de evaluar los conocimientos teóricos y el comportamiento de 353 pacientes; a pesar de que el $74 \%$ de ellos realizó correctamente el intercambio (dato similar al de nuestro estudio), consideró que el $47 \%$ necesitaban un reciclaje/refuerzo, siendo menor 
la proporción de pacientes con estudios universitarios ${ }^{7}$. Algo a destacar es que ha tenido en cuenta conocimientos teóricos y que la ejecución práctica se realizó en el propio domicilio.

Ambas cuestiones y el hecho de valorar a todos los pacientes en la realización de un intercambio manual, independientemente de la modalidad domiciliaria habitual, podría ser una limitación a nuestro estudio, sin embargo, no se han obtenido diferencias significativas como podríamos esperar. Si lo analizamos, tal vez, nos lleve a pensar que los pacientes con técnica manual, debido a la rutina de realizar el mismo proceso 3-4 veces diarias, puede llegar a una situación de confianza con respecto a las medidas de asepsia y no realizarlas correctamente.

Posteriormente Dong y Chen, volvieron a examinar el procedimiento del cambio de bolsa de sus pacientes, lo hicieron a los 6 meses desde el inicio de la DP domiciliaria y lo relacionaron con el primer episodio de peritonitis posterior. Los datos fueron preocupantes, encontraron, entre otros, que el $51 \%$ no se lavaban correctamente las manos y el $11 \%$ no usaban mascarilla, aunque sólo esto último se reveló como factor de riesgo independiente para el desarrollo de la infección peritoneal'.

Nuestros resultados sin embargo, aunque mejorables, son más alentadores en cuanto a la colocación de mascarilla, lavado de manos, desinfección y conexión-desconexión.

Recientemente se ha publicado el estudio de Gadola et al, que utiliza como herramienta de valoración una evaluación objetiva estructurada (OSA) de las habilidades prácticas de los pacientes (realización del intercambio y solución de eventualidades) para relacionarlas con las tasa de peritonitis del año anterior; en una fase posterior y tras introducir un nuevo programa de educación que consistía en clases individuales, talleres grupales y reciclajes volvió a registrar las infecciones peritoneales, que habían mejorado ${ }^{10}$. Sayed et al presenta resultados similares, poniendo de manifiesto la importancia de las reevaluaciones periódicas en el domicilio para disminuir las complicaciones infecciosas incluso en las condiciones más adversas ${ }^{11}$.

Todos estos estudios concluyen en la necesidad de realizar programas de reentrenamiento o refuerzo periódi$\mathrm{Co}^{12} \mathrm{y}$ así lo recogen nuestras guías ${ }^{13}$; en lo que no existe unanimidad es en el momento en el que deberíamos hacerlo; se señalan los 6 meses como punto clave, pero pocas veces se realizan de forma programada y dejan a criterio de la enfermera esta cuestión.

Parece lógico entonces, que se establezca un protocolo de reentrenamiento que incluya práctica y aspectos teóricos esenciales de la DP. Lo más riguroso sería hacerlo en el domicilio de cada paciente y con su técnica habitual para observarlos en su rutina y poder identificar y corregir las desviaciones en su ejecución.

La técnica de DP, sin duda, mejora la calidad de vida de los pacientes con ERC pero para ello es imprescindible un gran esfuerzo por parte de éstos y una gran dedicación por parte del personal de enfermería.

\section{Conclusiones}

Nuestros pacientes están, en general, bien formados, con variaciones individuales no dependientes de edad, sexo, educación o tiempo en la técnica.

Consideramos que un buen entrenamiento no es suficiente, siendo pertinente establecer un programa de reentrenamientos periódicos y personalizados.

\section{Agradecimientos}

Agradecemos la inestimable colaboración de nuestros pacientes que nos ayudan a mejorar cada día; sin ellos este trabajo no habría sido posible.

De igual manera, agradecer la colaboración de nuestros compañeros Nefrólogos, que nos apoyan y ayudan en cada nuevo proyecto que emprendemos.
Recibido: 8 Agosto 2013
Revisado: 12 Agosto 2013
Modificado: 21 Agosto 2013
Aceptado: 25 Agosto2013

\section{Bibliografía}

1. Castro M J, Sánchez S, Celadilla 0, Martínez M V. Enseñanza de las técnicas dialíticas peritoneales. En: Montenegro J, Olivares J. La diálisis peritoneal. España: Editorial Dibe; 1999: 181-199. 
2. Bajo M A, Vega N, González-Parra E. Estructura y necesidades de una Unidad de diálisis peritoneal. Nefrología 2006; 26 (4): 26-35.

3. Luongo $M$, Prowant B. Peritoneal Dialisys Program Organization and Management. The Nurse's Role. En: Nolph K D. Nolph and Gokal's Textbook of Peritoneal Dialysis. New York: Ed Springer; 2009: 335-370.

4. Barone R J, Campora M I, Giménez N S, Ramírez L, Santopietro M, Panese S A. The importance of the patient's training in chronic peritoneal dialysis and peritonitis. Advances in Peritoneal Dialysis 2011; 27: 97-100.

5. Bernardini J, Price V, Figueiredo A. ISPD Guidelines/Recommendations. Peritoneal dialysis patient training 2006. Peritoneal Dialysis International 2006; 26: 625-632.

6. Martín J L, Cirera F, Reina M. Formación proporcionada a los pacientes de diálisis peritoneal domiciliaria en España. Rev Soc Esp Enferm Nefrol 2008; 11 (1): 13-19.

7. Russo R, Manili L, Tiraboschi G, Amar K, De Luca M, Alberghini $E$, Ghiringhelli $P$ et al. Patient re-training in peritoneal dialysis: why and when it is needed. Kidney International 2006; 70: 127-132.
8. Bernardini J. Training and retraining: impact on peritonitis. Peritoneal Dialysis International 2010; 30: 434-436.

9. Dong J, Chen Y. Impact of the bag exchange procedure on risk of peritonitis. Peritoneal Dialysis International 2010; 30: 440-447.

10. Gadola L, Poggi C, Poggio M, Sáez L, Ferrari A, Romero $\mathrm{J}$ et al. Using a Multidisciplinary training program to reduce peritonitis in peritoneal dialysis patients. Peritoneal Dialysis International 2013; 33: 38-45.

11. Sayed S, Abu-Aisha H, Ahmed M, Elamin S.Effect of the patient's knowledge on peritonitis rates in peritoneal dialysis. Peritoneal Dialysis International 2013; 33: 362-366.

12. Gómez A C, Ojeda M A, Carballo E, Ramírez M A, Cárcamo J, Fernández D. Los indicadores del manejo del régimen terapéutico y su relación con la evolución de la información adquirida durante el entrenamiento en diálisis peritoneal. Rev Soc Esp Enferm Nefrol 2011; 14 (2): 83-89.

13. Granado A, Ruiz C, Arrieta J. Adiestramiento del paciente que se incorpora a diálisis peritoneal. Visitas domiciliarias. Reentrenamiento. Guías de práctica clínica en diálisis peritoneal. Nefrología 2006; 26(4): 57-66. 